\title{
EFFECTIVENESS OF THE TARGET ANTIBIOTIC ADMINISTRATION IN THE TREATMENT OF THE SEVERE CHRONIC PERIODONTITIS PART I - MICROBIOLOGICAL EVALUATION
}

\author{
K. Kotsilkov ${ }^{1}$, Chr. Popova ${ }^{1}$, L. Boyanova ${ }^{2}$, L. Setchanova ${ }^{2}$, G. Gergova ${ }^{2}$, T. \\ Kostyanev $^{2}$, D. Yordanov ${ }^{2}$, I. Mitov, V. Dosseva ${ }^{1}$ \\ 1. Department of Periodontology, Faculty of Dental Medicine, \\ 2. Department of Microbiology, Faculty of Medicine, \\ Medical University of Sofia, Bulgaria
}

\begin{abstract}
:
INTRODUCTION: The microbial etiology of inflammatory periodontal diseases provides the rationale for the use of antimicrobial medication in periodontal therapy. Scaling and root planning (SRP) is generally the first treatment employed for periodontitis. Antibiotics may be prescribed for periodontal patients who do not respond to conventional mechanical therapy, for patients with acute periodontal infections associated with systemic manifestations, for prophylaxis in medically compromised patients, and as an adjunct to surgical and non-surgical periodontal therapy.
\end{abstract}

AIM: The objects of the study are:

1. To determine the value of the microbiological diagnosis with culturing method in the treatment planning of the periodontal diseases.

2. To evaluate the importance of the susceptibility testing for the target antimicrobial treatment of periodontitis.

3. To evaluate the effectiveness of the adjunctive target antimicrobial administration in periodontitis cases.

MATERIALS AND METHODS: A total of 17 patients with aggressive or severe chronic periodontitis were included in this study. All patients must have at least 15 teeth and 4 periodontal sites with $\mathrm{PD}>4 \mathrm{~mm}$ in different quadrants of the dentition. Microbiological samples were taken from the periodontal pockets using paper points. The samples were taken from tree categories periodontal sites with PD 3-5mm, 5-7mm and $>7 \mathrm{~mm}$. All samples were analyzed with culture method. Selected colonies from the patients from the test groups were tested for antimicrobial susceptibility.

A standardized oral hygiene motivation program was applied to all patients. The Full Mouth Treatment approach was selected for therapy of the patients. Amoxicillin 500mg and Metronidazole 250mg tid for 10 days were administrated on the control group. The patients from the test group received antimicrobial treatment according to the results from the susceptibility analysis. Clinical, radiograph and microbiological evaluations are done again after 3 months.

RESULTS: The results from the microbiological analysis are presented in schemes.

CONCLUSION: The indiscriminate use of antimicrobials could be influencing the appearance of resistant strains associated with periodontal diseases in the population. That's why the use of antibiotics must be based on susceptibility testing, instead of a unique protocol of adjunctive antimicrobial regimen.

Key words: periodontal disease, microbiological analysis, antimicrobial therapy, antimicrobial susceptibility.

\section{INTRODUCTION:}

The microbial etiology of inflammatory periodontal diseases provides the rationale for the use of antimicrobial medication in periodontal therapy. As evidence for bacterial specificity in periodontitis has accumulated and strengthened over the past three decades, dentists have increased their use of systemic antibiotics in periodontal therapy. This concept is based on the premise that specific microorganisms cause destructive periodontal disease and that the antibiotic agent in vivo can exceed concentrations necessary to kill or inhibit the pathogen(s). Antibiotics are defined in this report as naturally occurring or synthetic organic substances that in low concentrations can inhibit or kill selective microorganisms. Antibiotics may be prescribed for periodontal patients who do not respond to conventional mechanical therapy, for patients with acute periodontal infections associated with systemic manifestations, for prophylaxis in medically compromised patients, and as an adjunct to surgical and non-surgical periodontal therapy. $(4,6,7,12)$

For the past 100 years, many investigations have attempted to define the etiologic agents of these diseases. The microbiology of periodontal infections is quite complicated, and numerous bacterial agents have been implicated in their etiology. Perhaps as much as 50 percent 
of the subgingival flora of chronic periodontitis has not yet been characterized. Nonetheless, small groups of specific bacterial species are now considered to be important in the initiation or progression (or both) of periodontitis. Often mentioned are Bacteroides forsythus, Porphyromonas gingivalis, Treponema denticola, and Actinobacillus actinomycetemcomitans $(1,4,5,12,13)$.

Scaling and root planning (SRP) is generally the first treatment employed for periodontitis. Scaling may be performed with hand instruments alone or with the aid of an ultrasonic scaler. It is done to clean teeth thoroughly below the gum line, removing bacterial plaque, calculus (tartar), debris, necrotic tissue, and pus from pockets that form around infected teeth. Root planning involves cleaning and smoothing the root surface of an infected tooth after scaling so that the gingival tissue can heal close to the root, shrinking the tissue and reducing the depth of the pocket that had formed. SRP is intended to reduce the bacterial load, shrink swollen and inflamed gingiva, and recondition the subgingival ecology, making it biologically compatible with optimal healing and reattachment of epithelium to the root surface $(1,7)$.

The infectious etiology of the periodontal diseases justifies the long use of antibiotics in the periodontal therapy. Today we know that the diseases caused from microbial biofilms, like the chronic periodontitis, are very difficult to treat. The routine use of antimicrobials in the treatment of the chronic periodontitis is questionable because of the enormously high resistance of the microorganisms in the biofilms, and the very difficult antibiotic penetration $(2,3,8-11,14-18)$.

\section{AIM:}

The objects of the study are:

1. To determine the value of the microbiological diagnosis with culturing method in the treatment planning of the periodontal diseases.

2. To evaluate the importance of the susceptibility testing for the target antimicrobial treatment of periodontitis.
3. To evaluate the effectiveness of the adjunctive target antimicrobial administration in periodontitis cases

This presentations reviews only the first part of the study

\section{MATERIALS AND METHODS:}

A total of 17 patients with aggressive or severe chronic periodontitis were included in this study. All patients must have at least 15 teeth and 4 periodontal sites with $\mathrm{PD}>4 \mathrm{~mm}$ in different quadrants of the dentition. Exclusion criteria were pregnancy, lactation, system antibiotic administration or periodontal therapy in the previous 3 months, and suffering from serious chronic medical conditions (e.g., diabetes mellitus, kidney or liver disease) or presenting with an acute systemic infection. The patients were divided in two groups by coin toss. Microbiological samples were taken from the periodontal pockets using paper points. The samples were taken from tree categories periodontal sites with PD 3-5mm, 5-7mm and $>7 \mathrm{~mm}$.

All samples were analyzed with culture method. Selected colonies from the patients from the test groups were tested for antimicrobial susceptibility to Tetracycline, Amoxicillin, Metronidazole, Clindamycin, Azitromycin, and Ciprofloxacin.

A standardized oral hygiene motivation program was applied to all patients. The Full Mouth Treatment approach was selected for the mechanical therapy of the patients. Amoxicilline 500mg and Metronidazole $250 \mathrm{mg}$ tid for 10 days were administrated on the patients from the control group. The patients from the test group received antimicrobial treatment according to the results from the susceptibility analysis. Clinical, radiograph and microbiological evaluations are done again after 3 months.

\section{RESULTS:}

The results from the culturing are presented in the following schemes: 
AEROBES:

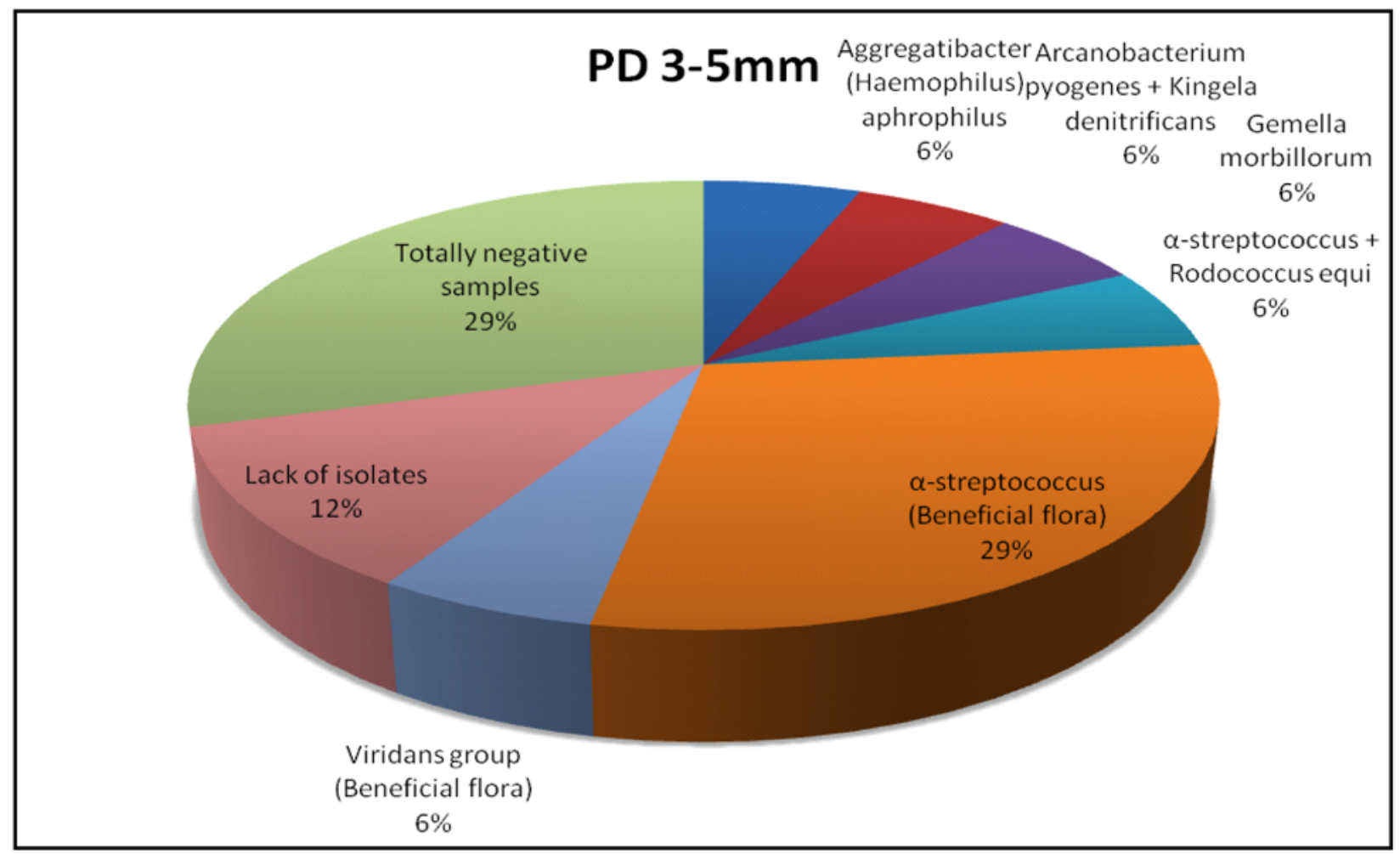

Fig. 1. Aerobic isolates from pockets with probing depth from 3-5mm.

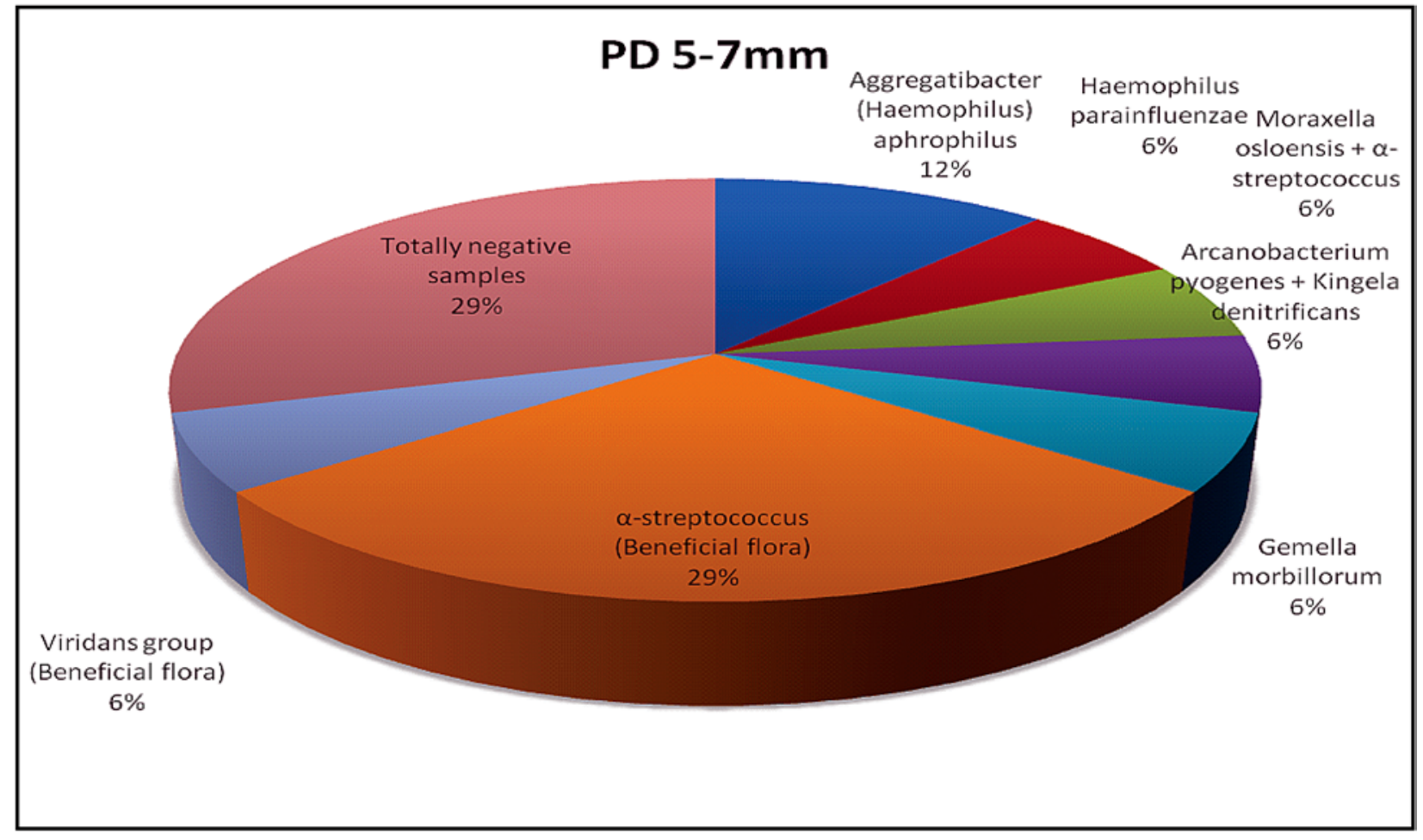

Fig. 2. Aerobic isolates from pockets with probing depth from 5-7mm. 


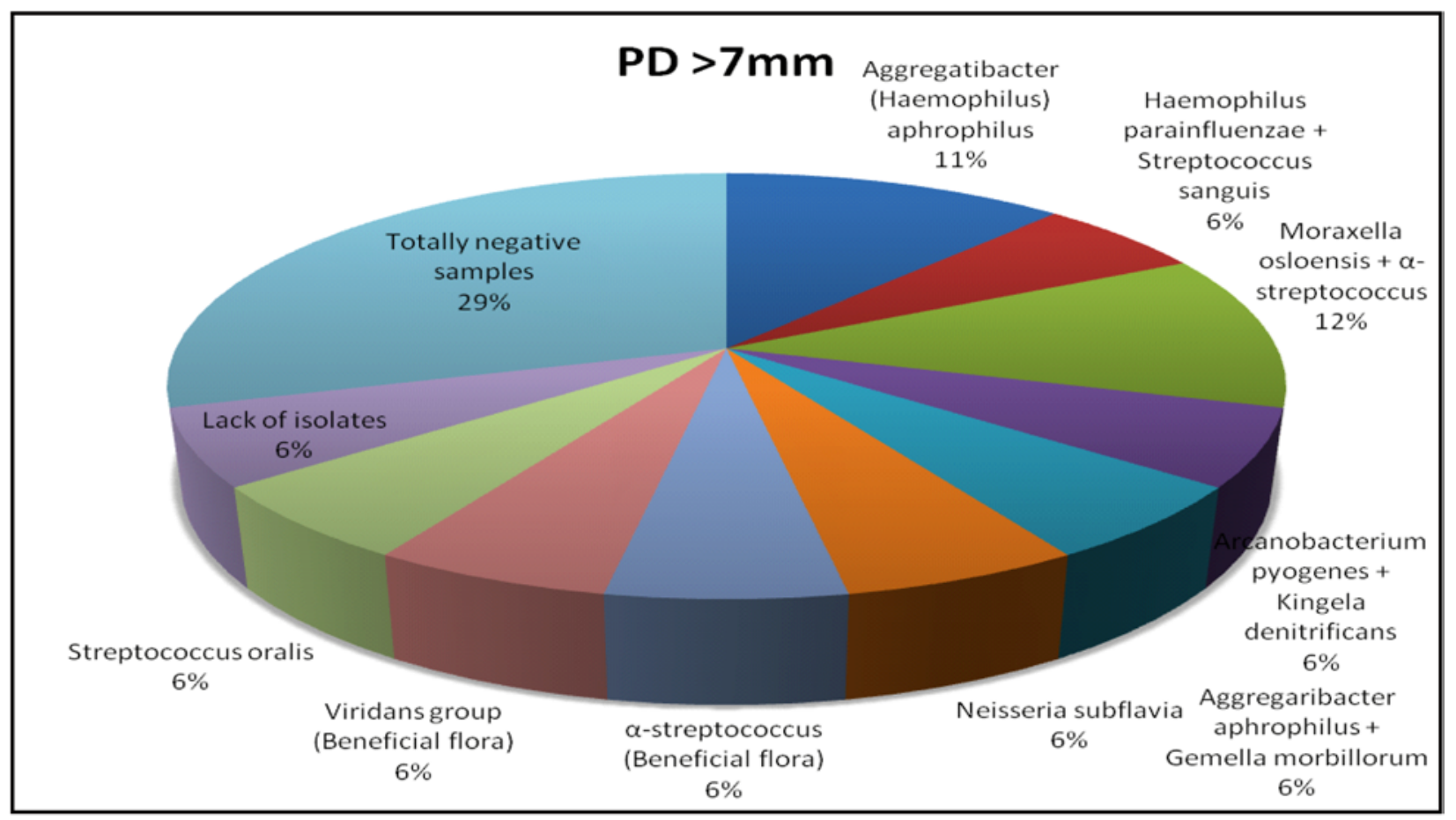

Fig. 3. Aerobic isolates from pockets with probing depth above $7 \mathrm{~mm}$.

In $6 \%$ of shalow periodntal pockets with PD $3-5 \mathrm{~m}$ the periodontal pockets presented aerobic isolates Agregatibacter aphrophilus has been detected. The percentage of this pathogen reises with the increase of the pocket depth $-12 \%$ in pockets with PD $5-7 \mathrm{~mm}$ and $11 \%$ with PD above $7 \mathrm{~mm}$. This results support the adjunctive antibiotic administration in patients with advanced periodontitis and prevalent pocket depth above $5 \mathrm{~mm}$.

\section{ANAEROBES}

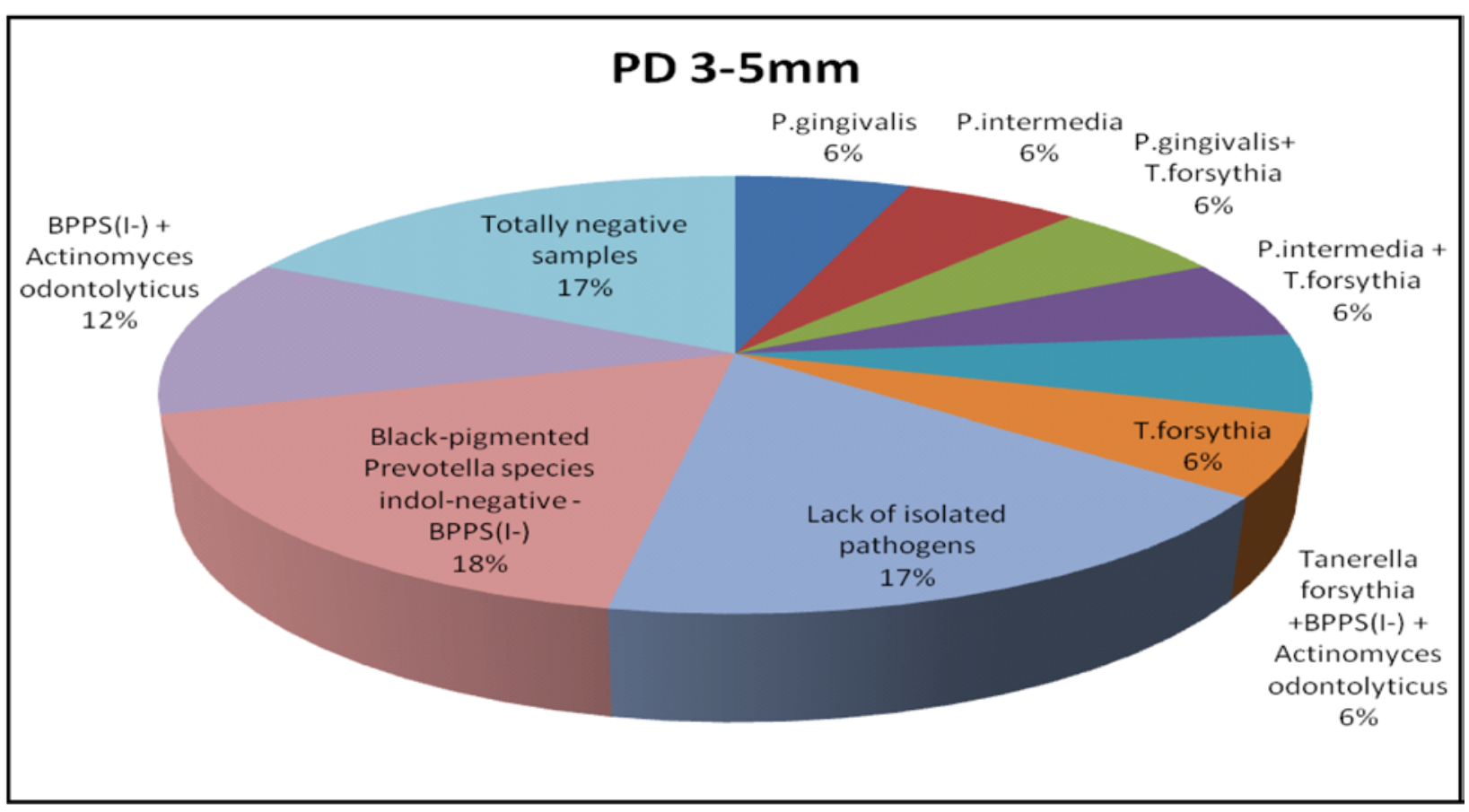

Fig. 4. Anaerobic isolates from pockets with probing depth from 3-5mm. 


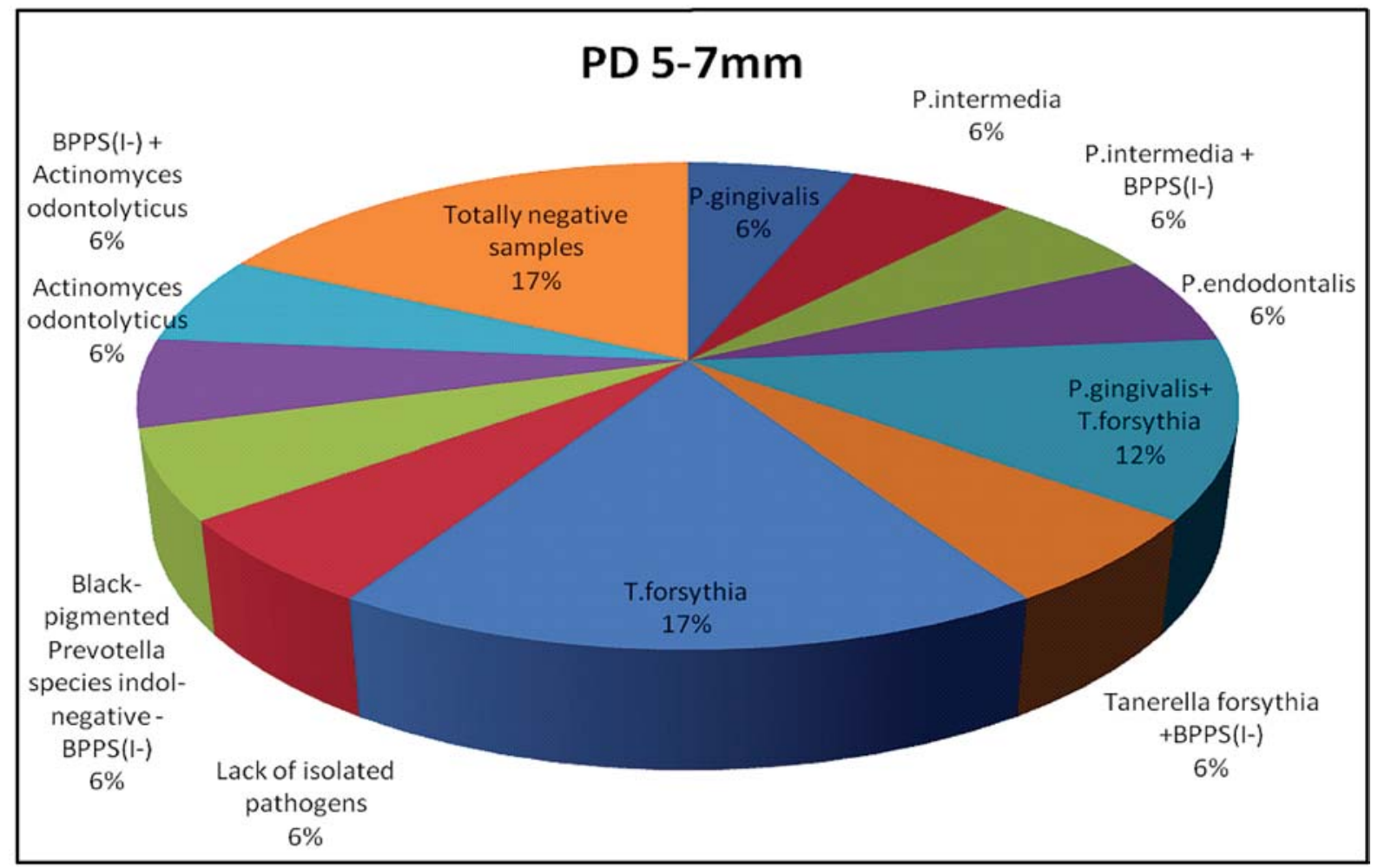

Fig. 5. Anaerobic isolates from pockets with probing depth from 5-7mm.

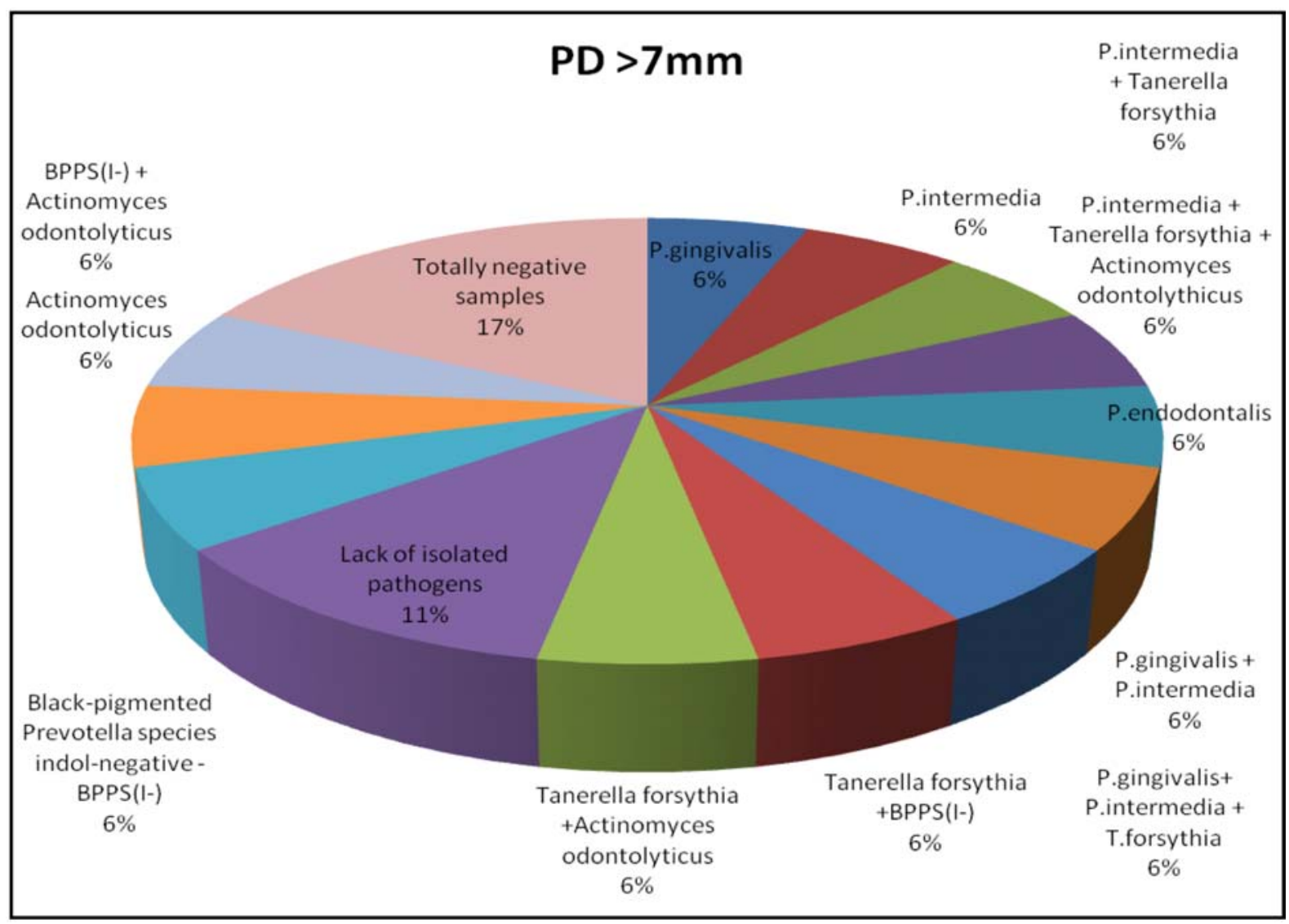

Fig. 6. Anaerobic isolates from pockets with probing depth above $7 \mathrm{~mm}$. 
The results from the cultivation of the subgingival isolates reveals increasing of the presence of perioidontopathogens with the increase of the probing depth. In the pockets with PD 3-5mm P.gingivalis and P.intermedia are isolated in $12 \%$ of the patients, T.forsythia in $24 \%$. The pockets with PD 5-7 $\mathrm{mm}$ have P.gingivalis in $18 \%$ of the patients, P.intermedia in $12 \%$ and T.forsythia in $35 \%$. The pockets with PD above $7 \mathrm{~mm}$ have P.gingivalis in $18 \%$, P.intermedia in $30 \%$ and T.forsytia also in $30 \%$ of the patients. With the increase of the probing depth the subgingival flora becomes more complex.In the tree groups of pockets P.gingivalis and P.intermedia are isolated as monoinfection in $6 \%$ of the patients. In the other patients a polyinfection is detected with the simultaneous presence of P.intermedia + Tanerella forsythia; Black-pigmented Prevotella species indol-negative - BPPS(I-) + T.forsytia; P.intermedia + BPPS(I-); P.gingivalis + T.forsythia; P.gingivalis + P.intermedia; P.intermedia + Tanerella forsythia + Actinomyces odontolythicus; The three periodontopatogens were simultaneously isolated only in the pockets with $\mathrm{PD}$ above $7 \mathrm{~mm}$ in $6 \%$ of the patients.

The increase of the presence and the complexity of the microbiota in the subgingival biofilm with the increasing of the probing depth advocates the administration of adjunctive antimicrobials in periodontitis patients with predominant pocket probing depth more than $5 \mathrm{~mm}$ to ensure the elinination of the pathogens from the subgingival region.

The results from the antimicrobial susceptibility analysis for the anaerobic species are presented in Table 1:

\begin{tabular}{|l|c|c|c|}
\hline $\begin{array}{l}\text { Resistance } \\
\text { of the isolates }\end{array}$ & P. gingivalis & P. intermedia & P. endodontalis \\
\hline Tetracycline & $33 \%$ & $38 \%$ & $0 \%$ \\
\hline Amoxicillin & $0 \%$ & $13 \%$ & $0 \%$ \\
\hline Metronidazole & $0 \%$ & $0 \%$ & $0 \%$ \\
\hline Clindamycin & $0 \%$ & $0 \%$ & $0 \%$ \\
\hline Azithromycin & $0 \%$ & $0 \%$ & $0 \%$ \\
\hline Ciprofloxacin & $0 \%$ & $0 \%$ & $0 \%$ \\
\hline $\begin{array}{l}\text { Amoxicillin and } \\
\text { Ciprofloxacine }\end{array}$ & $0 \%$ & $12,50 \%$ & $0 \%$ \\
\hline $\begin{array}{l}\text { Tetracycline and } \\
\text { Ciprofloxacin }\end{array}$ & $0 \%$ & $12,50 \%$ & $0 \%$ \\
\hline
\end{tabular}

Table 1. Antimicrobial resistance of the anaerobic isolates.
The data from the antimicrobial susceptibility reveals significant percent resistance to Tetracycline (33\% and 38\% accordingly) and $13 \%$ of the P. intermedia isolates are resistant to Amoxicillin. Multiple resistances have been detected only for P. intermedia strains - $12.5 \%$ of them were resistant to Amoxicillin and Ciprofoloxacin and $12.5 \%$ to Tetracycline and Ciprofloxacin. Monoresistance to Ciprofloxacin was not detected in the presented study. These results suggest that for improvement of the efficacy of the nonsurgical therapy in advanced periodontitis cases is appropriate to choose the antimicrobial agent after susceptibility testing. This approach could limit the need for surgical procedures in the periodontitis treatment.

\section{CONCLUSION:}

The adjunctive administration of systemic antibiotics could contribute to better control of the periodontal disease in cases with severe chronic or aggressive periodontitis. This approach could lead to less frequent recall visits and limit the need for periodontal surgery.

The indiscriminate use of antimicrobials could be influencing the appearance of resistant strains associated with periodontal diseases in the population. That's why the use of antibiotics must be based on susceptibility testing, instead of a unique protocol of adjunctive antimicrobial regimen. 


\section{REFERENCES:}

1. Bonito A.J., Lohr K.N., Lux L., Sutton S., Jackman A., Whitener L., Evensen C. Effectiveness of Antimicrobial Adjuncts to Scaling and Root Planing Therapy for Periodontitis. Volume 1. Evidence Report and Appendixes.AHRQ Publication No. 04-E014-3 March 2004

2. Ellen R.P., McCulloch C.A.G. Evidence versus empiricism: Rational use of systemic antimicrobials for treatment of periodontitis. Periodontol 2000 1996; 10: 29-44.

3. Haffajee A.D., Socransky S.S., Gunsolley J.C. Systemic anti-infective periodontal therapy. A systematic review. Ann Periodontol 2003;8:115-181

4. Haffajee A.D., Socransky S.S. Microbial etiological agents of destructive periodontal diseases. Periodontol 2000 1994; 5:78-111.

5. Listgarten M.A., Loomer P.M. Microbial identification in the management of periodontal diseases: A systematic review. Ann Periodontol 2003;8:182-192.

6. Loesche W. J., Grossman N. S.. Periodontal Disease as a Specific, albeit Chronic, Infection: Diagnosis and Treatment. Clin Microbiol Rev. 2001
October; 14(4): 727-752.

7. Position Paper Systemic Antibiotics in Periodontics J Periodontol 2004; 75: 1553-1565

8. Ramberg P., Rosling B., Serino G., Hellstrom M.K., Socransky S.S., Lindhe J. The long-term effect of systemic tetracycline used as an adjunct to nonsurgical treatment of advanced periodontitis. J Clin Periodontol 2001; 28: 446-452.

9. Roberts M.C. Antibiotic toxicity, interactions and resistance development. Periodontol 2000 2002;28:280-297.

10. Slots J., Ting M. Systemic antibiotics in the treatment of periodontal disease. Periodontol 2000 2002;28:106-176.

11. Smith S.R., Foyle D.M., Daniels J., et al. A double-blind placebo-controlled trial of azithromycin as an adjunct to nonsurgical treatment of periodontitis in adults: Clinical results. J Clin Periodontol 2002; 29:54-61.

12. Socransky S.S., Haffajee A.D. Dental biofilms: Difficult therapeutic targets. Periodontol 2000 2002;28:12-55.

13. Socransky S.S., Haffajee A.D. Microbial mechanisms in the pathogenesis of destructive periodontal diseases: a critical assessment. J Periodont Res, 1991:26: 195-209

14. van Winkelhoff A.J., Herrera Gonzales D., Winkel E.G., DellemijnKippuw N., Vandenbroucke-Grauls C.M., Sanz M. Antimicrobial resistance in the subgingival microflora in patients with adult periodontitis. A comparison between The Netherlands and Spain. J Clin Periodontol 2000; 27:79-86.

15. van Winkelhoff A.J., Rams T.E., Slots J. Systemic antibiotic therapy in periodontics. Periodontol 2000 1996;10:4578.

16. Walker C., Karpinia K. Rationale for use of antibiotics in periodontics. J Periodontol 2002;73:1188-1196.

17. Walker C.B. The acquisition of antibiotic resistance in the periodontal flora. Periodontol 2000 1996;10:78-88.

18. Winkel E.G., van Winkelhoff A.J., Timmerman M.F., Van der Velden U., van der Weijden G.A. Amoxicillin plus metronidazole in the treatment of adult periodontitis patients. A double-blind placebocontrolled study. J Clin Periodontol 2001;28:296-305.

\author{
Address for correspondence: \\ Dr K. Kotsilkov \\ Department of Periodontology, Faculty of Dental Medicine, \\ Medical University of Sofia, \\ 1, Georgi Sofiiski Str., Sofia, Bulgaria \\ Mobile: +359898781299 \\ E-mail: kotsilkov@mail.bg
}

